\title{
Calculation of Groundwave Attenuation in the Far Diffraction Region
}

\author{
L. E. Vogler \\ Contribution From the Central Radio Propagation Laboratory, National Bureau of Standards, \\ Boulder, Colo.
}

(Received January 3, 1964)

\begin{abstract}
This paper presents a graphical method of determining the groundwave attenuation over a spherical homogeneous earth in the far diffraction region. The curves are applicable to either vertical or horizontal polarization and to any combination of effective earth's radius, electromagnetic ground constants, frequency, path distance, and antenna heights. A criterion is given that indicates the method may be used not only for far beyond line-ofsight paths but, in many practical situations, at line-of-sight or even slightly within. Examples illustrating the use of the formulas and curves are included.
\end{abstract}

\section{Introduction}

In a previous paper [Vogler, 1961] a simplified graphical method was presented for calculating smooth homogeneous earth groundwave attenuation in the far diffraction region assuming horizontal polarization of the radio waves. In the present paper this method will be extended to include the case of vertical polarization.

The far diffraction region is defined here as that region in which the diffracted field intensity may be determined by the first term of the Van der PolBremmer residue series [Bremmer, 1949]. This region extends from near the radio horizon to all greater distances. In some situations the first term provides a valid approximation to the diffracted field even at points slightly within line-of-sight, and a criterion for determining the minimum distance is given in section 2 .

Since this paper is meant as a practical aid in obtaining diffraction calculations, no mathematical derivation of the functions discussed later is included. Detailed explanations of the residue series are given in the work by Bremmer [1949] and also in the introduction to the CCIR Atlas [Atlas, 1955]. As far as possible, the notation used is that established by Norton [1941] when identical quantities are considered; e.g., the parameters $K$ and $b^{\circ}$. The three functions $C_{1}, F$, and $G$ from which the attenuation is calculated were defined in such a manner as to facilitate graphical interpolation for any combination of frequency and distance. Numerical evaluations of these functions were obtained through the use of an electronic computer.

\section{Groundwave Attenuation}

The attenuation $A_{t}$ (measured in decibels) relative to a free space inverse distance field (see, for example,
(18)), may be divided into four terms containing, essentially, the distance dependence, two antenna height dependences, and a term depending on the electric constants of the ground:

$$
A_{t}=G\left(x_{0}\right)-F\left(x_{1}\right)-F\left(x_{2}\right)-C_{1}\left(K, b^{\circ}\right),
$$

where

$$
x_{0,1,2}=\beta_{0} C_{0}^{2} f_{\mathrm{MHz}}^{\frac{1}{3}} d_{0,1,2}, C_{0}=(4 / 3 k)^{\frac{1}{3}},
$$

and $f_{\mathrm{MHz}}$ denotes the radiofrequency in megahertz. $d_{0}$ is the total arc distance between antennas, and $d_{1}$ and $d_{2}$ are distances from the transmitting and receiving antennas to their radio horizons (see fig. 1). For antenna heights $h_{1}$ and $h_{2}$ not too large, the horizon distances may be approximated for a linear atmosphere with no ducts by

$$
d_{1,2} \simeq \sqrt{2(k a) h_{1,2}}
$$

where $k a$ is the effective radius of the sphere.

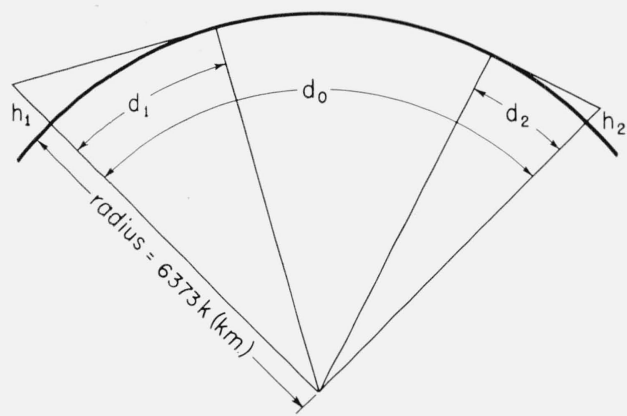

Figure 1. The geometry for smooth earth diffraction. 
In practical applications the factor $k$ is used to account approximately for the effects of atmospheric refraction [Burrows and Atwood, 1949] and is defined in terms of the gradient with respect to height, $d n / d h$, of the atmosphere's refractive index $n$ :

$$
k=\left[1+\left(\frac{a}{n} \frac{d n}{d h}\right)_{h=0}\right]^{-1} .
$$

It is usual to assume a standard " $4 / 3$ earth" atmosphere in presenting diffraction curves [Atlas, 1955; Wait and Howe, 1956], and this procedure is followed here. Thus, the $C_{0}$ appearing in (2) is merely a normalizing factor which for the standard atmosphere $(k=4 / 3)$ is equal to unity. Choosing a reference radius $a=6373 \mathrm{~km}$, (3) may now be expressed as

$$
d_{1,2}(\mathrm{~km}) \simeq 4.1225 \sqrt{C_{0}^{-3} h_{1,2}(\mathrm{~m})},
$$

where $h$ is in meters. The quantities $x_{0}, x_{1}$, and $x_{2}$ defined by (2) can be considered as parametric distances; $x_{0}$ referring to the total separation between antennas, and $x_{1}, x_{2}$ referring to the two antenna radio horizon distances.

The parameters $\beta_{0}, K$, and $b^{\circ}$ in (1) and (2) depend on the polarization of the wave and the relative dielectric constant $\epsilon$ and conductivity $\sigma$ of the ground. Assuming a homogeneous ground in which $\epsilon$ and $\sigma$ are everywhere constant, $K$ and $b^{\circ}$ are defined as follows for the two cases of vertical and horizontal polarization. Vertical polarization:

$$
\begin{gathered}
K_{v}=1.7778 \times 10^{-2} C_{0} f_{\mathrm{MHz}}^{-1 / 3}\left[\frac{\epsilon^{2}+s^{2}}{\sqrt{(\epsilon-1)^{2}+s^{2}}}\right]^{1 / 2}, \\
b_{v}^{\circ}=2 \tan ^{-1}\left(\frac{\epsilon}{s}\right)-\tan ^{-1}\left(\frac{\epsilon-1}{s}\right)
\end{gathered}
$$

horizontal polarization:

$$
\begin{gathered}
K_{h}=1.7778 \times 10^{-2} C_{0} f_{\mathrm{MHz}}^{-1 / 3}\left[(\epsilon-1)^{2}+s^{2}\right]^{-1 / 4}, \\
b_{h}^{\circ}=180^{\circ}-\tan ^{-1}\left(\frac{\epsilon-1}{s}\right),
\end{gathered}
$$

where $s$ depends on the ground conductivity in mhos per meter and radiofrequency, and is defined as

$$
s=1.8 \times 10^{4} \sigma(\operatorname{mhos} / \mathrm{m}) / f_{\mathrm{MHz}} .
$$

Note that for $\sigma(\operatorname{mhos} / \mathrm{m}) / f_{\mathrm{MHz}}>>(\epsilon / 2) \times 10^{-4}$ :

$$
\begin{gathered}
K_{v} \sim 2.385 C_{0} \sqrt{\sigma} / f_{\mathrm{MHz}}^{5 / 6}, b_{v}^{\circ} \sim 0, \\
K_{h} \sim 1.325 \times 10^{-4} C_{0} f_{\mathrm{MHz}}^{1 / 6} / \sqrt{\sigma}, b_{h}^{\circ} \sim 180 ;
\end{gathered}
$$

and for $\sigma(\mathrm{mhos} / \mathrm{m}) / f_{\mathrm{MHz}}<<(\epsilon / 2) \times 10^{-4}$ :

$$
\begin{aligned}
& K_{v} \simeq 1.7778 \times 10^{-2} C_{0} \epsilon / \sqrt{\epsilon-1} f_{\mathrm{MHz}}^{1 / 3}, b_{v}^{\circ} \simeq 90, \\
& K_{h} \simeq 1.7778 \times 10^{-2} C_{0} / \sqrt{\epsilon-1} f_{\mathrm{MHz}}^{1 / 3}, b_{h}^{\circ} \simeq 90 .
\end{aligned}
$$

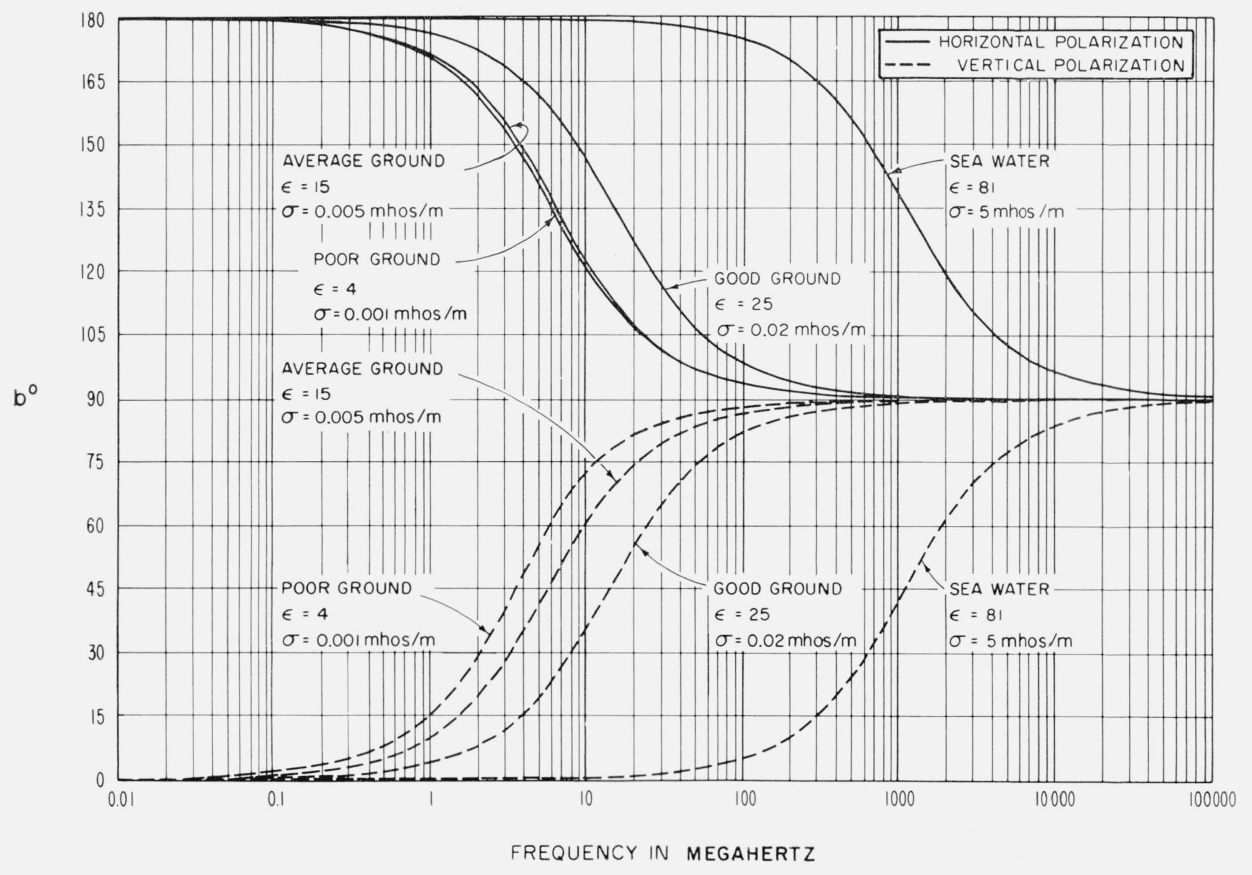

Figure 2. The parameter $\mathrm{b}_{\mathrm{v}}^{\circ}$ or $\mathrm{b}_{\mathrm{h}}^{\circ}$. 


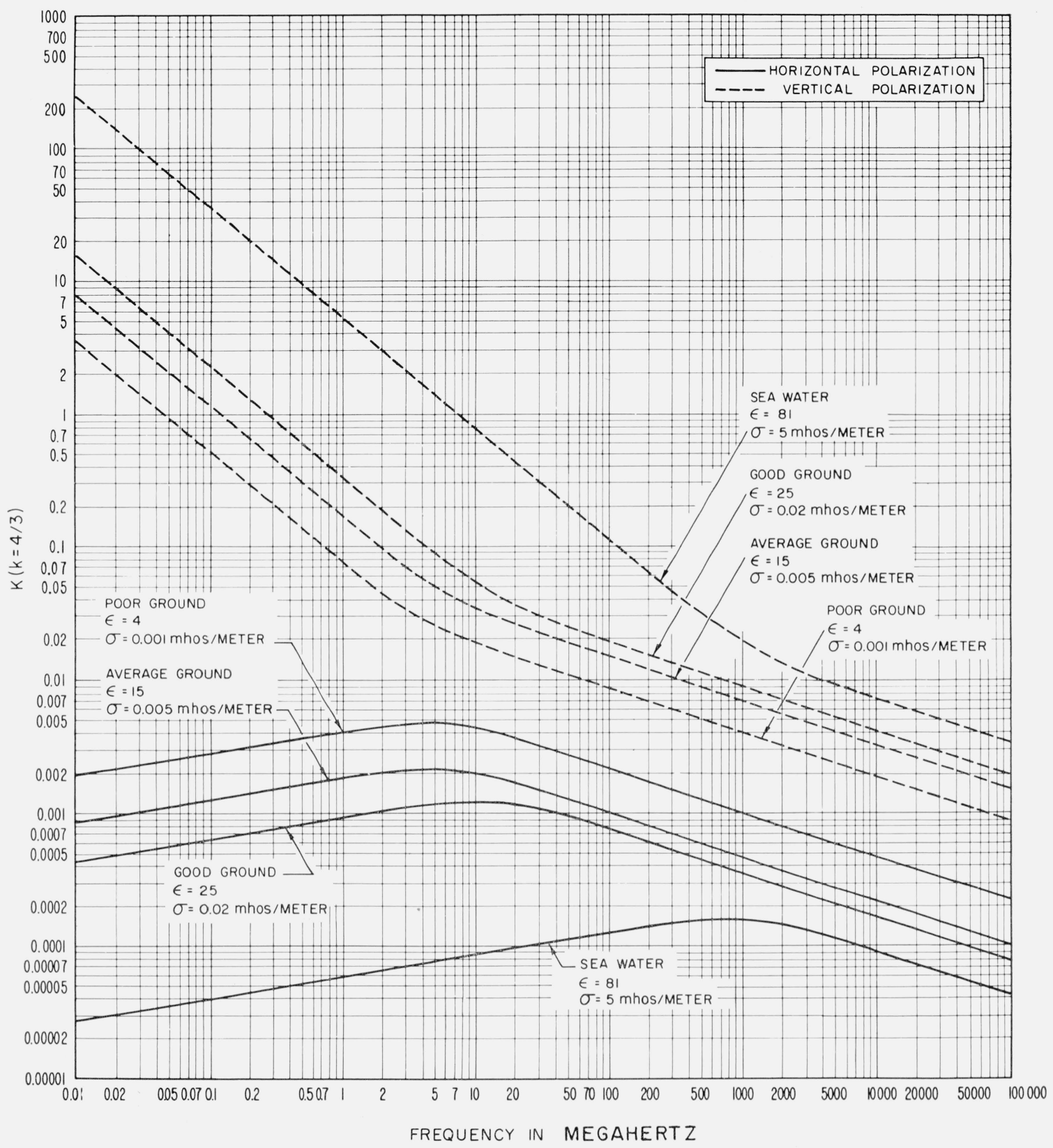

Figure 3. The parameter $\mathbf{K}_{v}$ or $\mathbf{K}_{\mathrm{h}}$. For general $k$, $K(k)=C_{0} K(4 / 3)$.

Curves of $b^{\circ}$ and $K$ versus $f_{\mathrm{MHz}}$ are shown in figures 2 and 3 for various combinations of $\epsilon$ and $\sigma$ corresponding to poor ground, average ground, good ground, and sea water. A value of $k=4 / 3$ was assumed in obtaining the curves of figure 3 ; however, they may be used for other $k$ through the simple relationship: $K(k)=C_{0} K(4 / 3)$.

The parameter $\beta_{0}$ appearing in (2) is plotted as a function of $K$ and $b^{\circ}$ in figure 4 . The limiting value of $\beta_{0}=1.607$ for $K \leqslant 0.01$ may be used for almost all practical cases of horizontal polarization. On the other hand for vertical polarization $\beta_{0}$ may range from 1.607, corresponding to high frequency propagation, down to 0.700 , corresponding to low frequency propagation over sea water. (See fgs. 3 and 4.)

With $K, b^{\circ}$, and $\beta_{0}$ evaluated, the groundwave attenuation may now be calculated by (1) and figures 5 and $7 . C_{1}\left(K, b^{\circ}\right)$, obtained from figure 5 , has the limiting values $C_{1}\left(0, b^{\circ}\right)=20.03$ for $K \rightarrow 0$ and $C_{1}\left(\infty, b^{\circ}\right)=20.94$ for $K \rightarrow \infty$; for any value of $K$ 


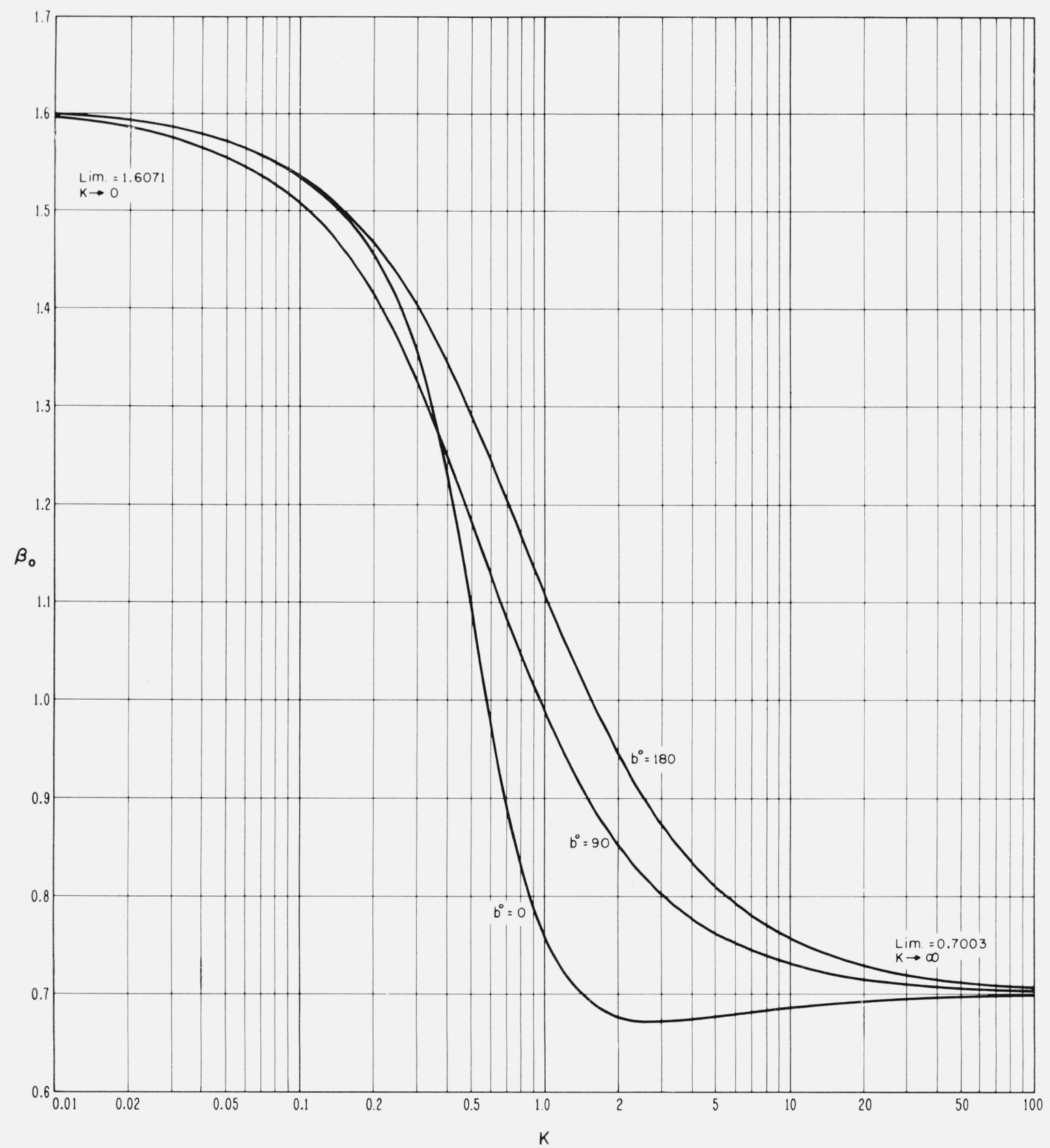

Figure 4. The parameter $\beta_{0}$.

it can be seen that $C_{1}$ does not vary more than about $6 \mathrm{~dB}$.

The functions $G\left(x_{0}\right), F\left(x_{1}\right)$, and $F\left(x_{2}\right)$ are shown in figure $7, G\left(x_{0}\right)$ being the uppermost curve and defined by:

$$
G\left(x_{0}\right) \equiv 5.751 \times 10^{-2} x_{0}-10 \log x_{0} .
$$

In general the height function $F(x)$ depends on $K$ and $b^{\circ}$, except for large values of $x_{1}$ or $x_{2}$, in which case $F(x) \sim G(x)$. For $x_{1}$ or $x_{2}$ approaching zero, the limiting values of $F(x)$ are plotted in figure 6 which may thus be used to help interpolate for those values of $K$ and $b^{\circ}$ not shown in figure 7 .

Since (1) is based upon only the first term of the residue series, a fairly good indication of its range of validity may be gained from the ratio of the second term $T_{2}$ to the first term $T_{1}$ of the series. If we require the error in $A_{t}$ to be less than some given 


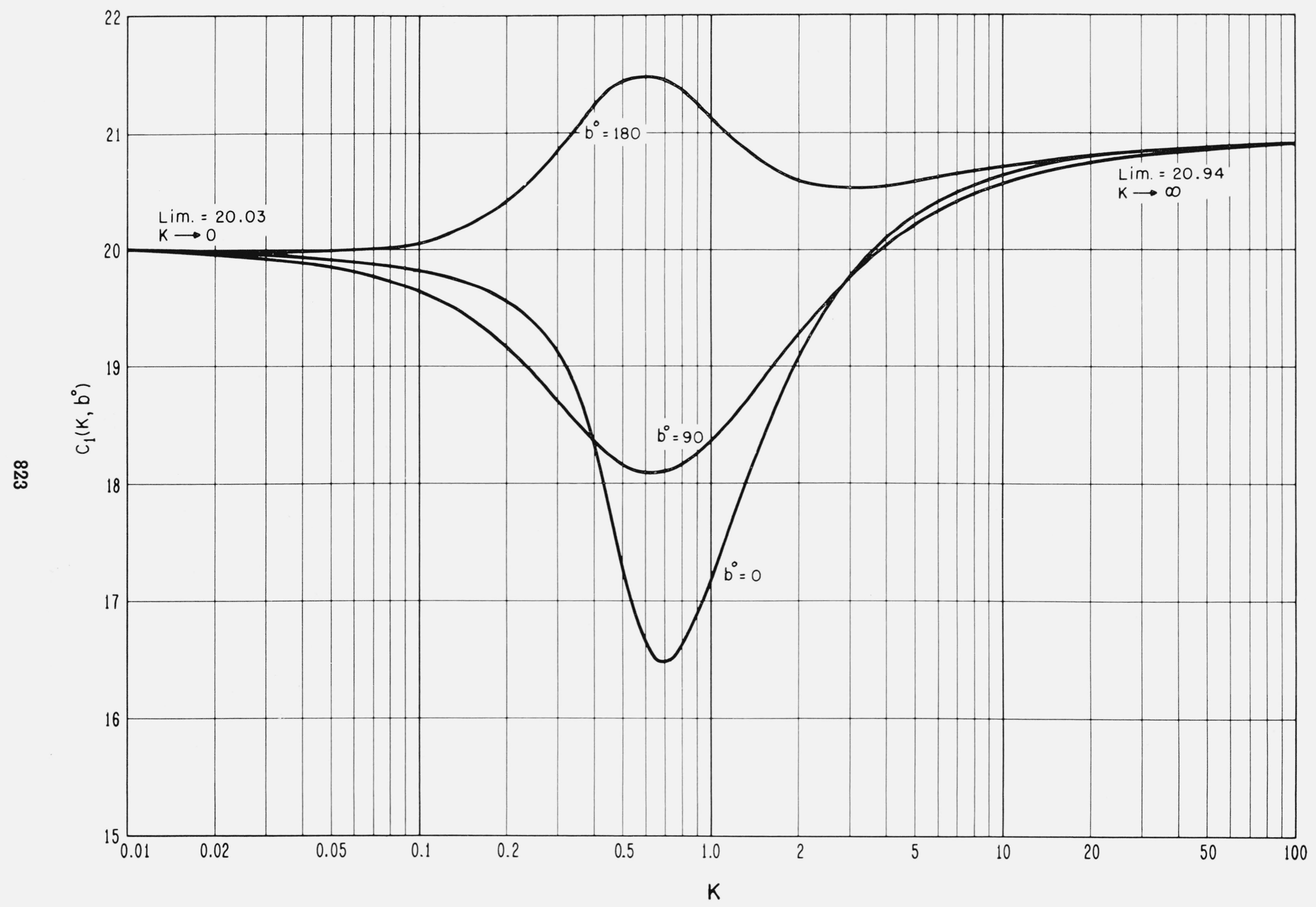

Figure 5. The term $\mathrm{C}_{1}\left(\mathrm{~K}, \mathrm{~b}^{\circ}\right)$ for use in (1). 


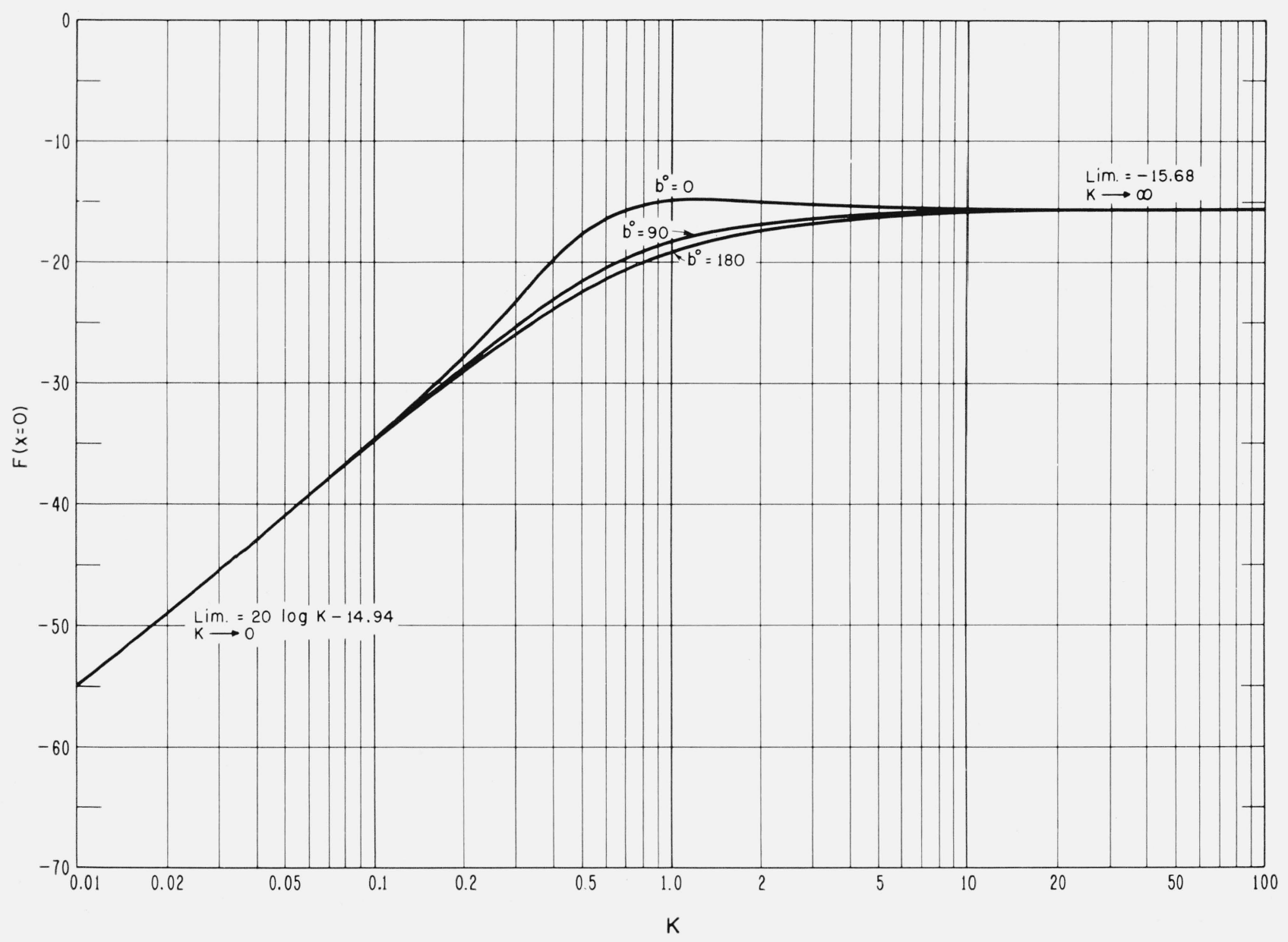

FIGURE 6. The height gain function $\mathrm{F}(\mathrm{x}=0)$ for zero antenna heights.

value, say $\delta$, we have that:

$$
20 \log \left|1+T_{2} / T_{1}\right| \leq 20 \log \left[1+\left|T_{2} / T_{1}\right|\right]<\delta .
$$

This is the procedure used to obtain the $\Delta\left(x_{1,2}\right)$ curves shown in the lower right hand corner of figure 7. Using the limiting values of $\beta_{0}(1.607$ and 0.700 ), then for $A_{t}$ to be accurate to within $1.5 \mathrm{~dB}$ (approximately) it may be shown that:

$x_{0}-x_{1} \Delta\left(x_{1}\right)-x_{2} \Delta\left(x_{2}\right)>335,\left(\beta_{0}=1.607, K \lesssim 0.1\right)$

$x_{0}-x_{1} \Delta\left(x_{1}\right)-x_{2} \Delta\left(x_{2}\right)>115,\left(\beta_{0}=0.700, K \gtrsim 10\right)$.

Notice that in certain cases (1) provides a good approximation to the attenuation even for just within line-of-sight paths as long as the appropriate condition (15a) or (15b) holds. For values of $\beta_{0}$ lying between the two limits, linear interpolation between the $\Delta(x)$ curves of figure 7 and the two minimum values of (15a) and (15b) will give a fair approximation to the range of validity of (1).
Using linear interpolation:

$$
x_{0}-x_{1} \Delta\left(x_{1}, \beta_{0}\right)-x_{2} \Delta\left(x_{2}, \beta_{0}\right)>x_{\min },
$$

where

$$
\begin{aligned}
x_{\min }=335-242.6\left(1.607-\beta_{0}\right), \\
\left.\begin{array}{rl}
\Delta\left(x, \beta_{0}\right)= & \Delta(x, 1.607)+1.103\left(1.607-\beta_{0}\right) \\
& \times\{\Delta(x, 0.700)-\Delta(x, 1.607)\} ;
\end{array}\right\}
\end{aligned}
$$

$\Delta(x, 0.700)$ and $\Delta(x, 1.607)$ are the values read from the upper and lower curves respectively in figure 7 .

\section{Examples}

In order to use (1) to calculate the field strength $E$ in a particular situation, a reference field $E_{0}$ must be specified. The relationship between $A_{t}$ and $E$ is then simply:

$$
E=E_{0}-A_{t}
$$




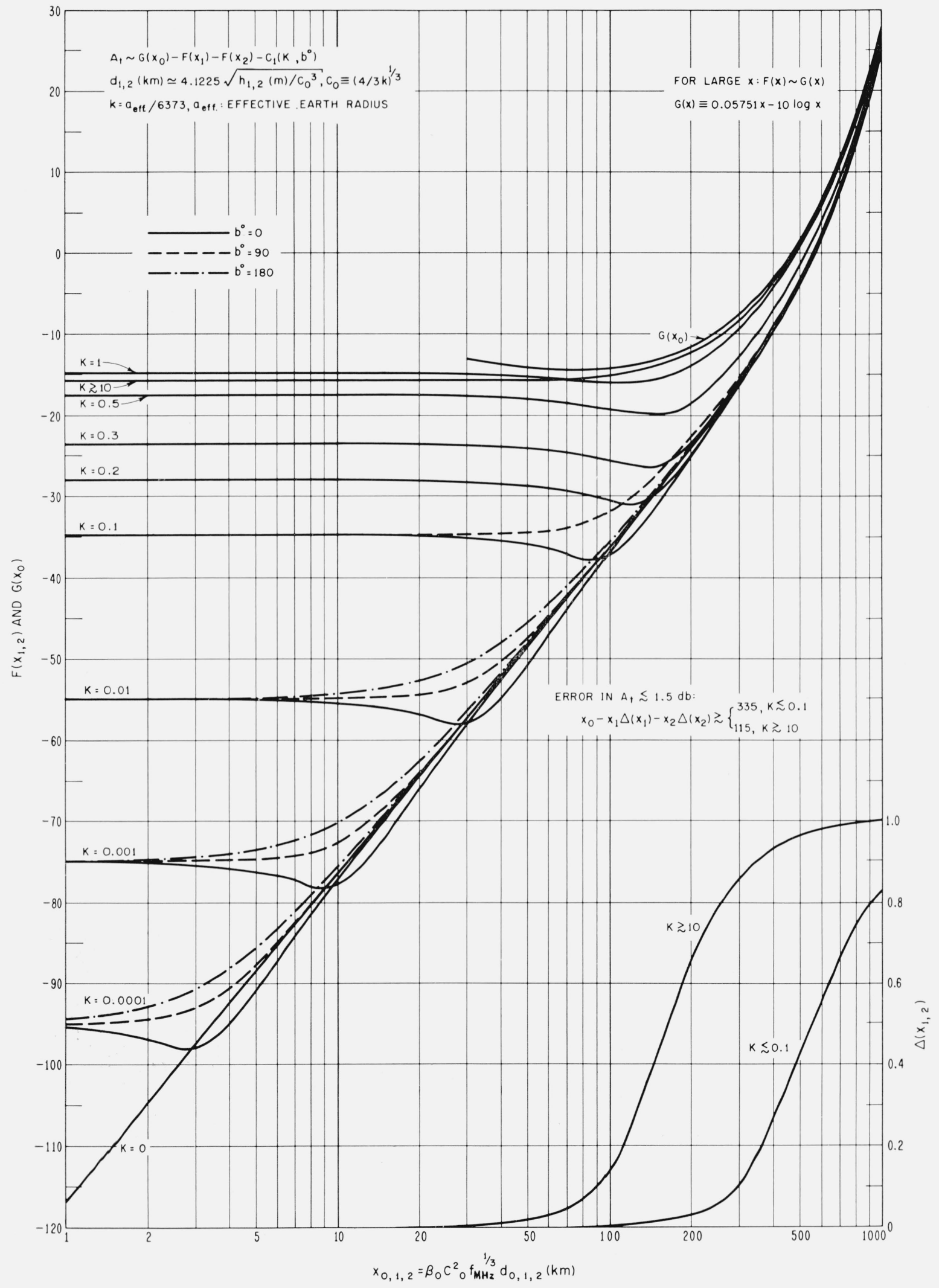

FIGURE 7. The distance function $\mathrm{G}\left(\mathrm{x}_{0}\right)$ and height function $\mathrm{F}\left(\mathrm{x}_{1,2}\right)$ for use in $(1)$; also $\Delta(\mathrm{x})$ for use in (15) or (16). 
For instance, the reference field (in $\mathrm{dB}$ above $1 \mu \mathrm{V} / \mathrm{m}$ ) assumed for the curves presented in the CCIR Atlas [At]as, 1955] is

$$
\begin{aligned}
E_{0}=20 \log \left[2.22 \times 10^{5} / d_{0}(\mathrm{~km})\right] & \\
& =106.93-20 \log d_{0}(\mathrm{~km}) .
\end{aligned}
$$

The following examples are given to illustrate the use of (1) in calculating field strength.

Given a particular propagation path in which $k=4 / 3, \epsilon=80, \sigma=4 \operatorname{mhos} / \mathrm{m}, f_{\mathrm{MHz}}=30, d_{0}=160 \mathrm{~km}$, $h_{1}=100 \mathrm{~m}$, and $h_{2}=1000 \mathrm{~m}$, then from $(5), d_{1}=41.225$ $\mathrm{km}$ and $d_{2}=130.365 \mathrm{~km}$; this corresponds to a within line-of-sight path. The fields for both vertical and horizontal polarization are then calculated as follows:

(1) Vertical polarization. From (9) the parameters $K_{v}$ and $b_{v}^{\circ}$ are calculated as $K_{v} \sim 0.28, b_{v}^{\circ} \sim 0$, and from the curves of figures 4 and $5, \beta_{0}=1.38$, $C_{1}(0.28,0)=19.2$. From $(2)$ the $x$ 's are calculated as $x_{0}=686, x_{1}=177, x_{2}=559$, and the criterion given by (16) indicates that (1) may be used in the present situation:

$686-177(0.16)-559(0.62)$

$$
=311>280 \text {, from }(16 \mathrm{a}) \text { and }(16 \mathrm{~b}) \text {. }
$$

Then from (1) and figure 7,

$$
A_{t}=11.0+25.5+0.5-19.2=17.8 \text {. }
$$

To compare with the CCIR Atlas, (17) and (18) are used giving the field strength as

$$
E=62.8-17.8=45.0(\mathrm{~dB} \text { above } 1 \mu \mathrm{V} / \mathrm{m}) .
$$

The value as read from the Atlas is $E=44.4$.

(2) Horizontal polarization. From (10) and figures 4 and $5, K_{h} \sim 1.2 \times 10^{-4}, b_{h}^{\circ} \sim 180, \beta_{0}=1.607$, $C_{1}\left(10^{-4}, 180\right)=20.0$; from (2) $x_{0}=799, x_{1}=206$, $x_{2}=651$, and from (1) and figure 7 ,

$$
A_{t}=17.0+23.5-4.5-20.0=16.0 \text {. }
$$

Equations (17) and (18) give $E=62.8-16.0=46.8$, whereas the value as read from the Atlas is $E=46.0$.

Radio propagation studies are often presented in terms of the propagation loss $L_{p}$ of a radio system [Norton, 1959; Wait, 1959]. The relationship between attenuation and propagation loss is

$$
L_{p}=32.45+20 \log f_{\mathrm{MHz}} d_{0}(\mathrm{~km})+A_{t}-G_{p},
$$

where $G_{p}$ denotes the path antenna gain which, for negligible polarization coupling loss, is just the sum of the free space antenna power gains above an isotropic antenna.

The system loss $L_{s}$ of a radio communication system is defined as the decibel ratio of the power input to the terminals of the transmitting antenna $P_{t}$ and the resultant power available at the terminals of the receiving antenna $P_{a}$ :

$$
L_{s}=P_{t}-P_{a} .
$$

Propagation loss and the practicably measurable system loss are then related by

$$
L_{s}=P_{\iota}-P_{u}=L_{p}+L_{t}+L_{r}
$$

where $L_{t}$ (transmitter) and $L_{r}$ (receiver) are the decibel ratios of the actual antenna input resistances $r$ to their free space radiation resistances $r_{f}$ :

$$
L_{t, r}=10 \log \left(r / r_{f}\right) .
$$

Curves of $L_{t}$ and $L_{r}$ for electric dipoles and small loop antennas without ground screens over finitely conducting ground are available in the literature [Vogler and Noble, 1963]. The effects of ground screens are considered in papers by Wait and Surtees [1954] and by Wait [1956].

\section{References}

Atlas of ground-wave propagation curves for frequencies between $30 \mathrm{Mc} / \mathrm{s}$ and $300 \mathrm{Mc} / \mathrm{s}$ (1959), (published by the International Telecommunications Union, Geneva).

Bremmer, H. (1949), Terrestrial radio waves (Elsevier Publishing Co., Amsterdam).

Burrows, C. R., and S. S. Atwood (1949), Radio wave propagation (Academic Press Inc., New York, N.Y.).

Norton, K. A. (1941), The calculation of ground-wave field intensity over a finitely conducting spherical earth, Proc. IRE 29, 623.

Norton, K. A. (July-Aug. 1959), System loss in radio wave propagation, J. Res. NBS 63D (Radio Prop.), No. 1, 53-73.

Vogler, L. E. (July-Aug. 1961), Smooth earth diffraction calculations for horizontal polarization, J. Res. NBS 65D (Radio Prop.), No. 4, 397-399.

Vogler, L. E., and J. L. Noble (May, 1965), Curves of ground proximity loss for dipole antennas, NBS Tech. Note 175.

Wait, J. R. (1956), Effect of the ground screen on the field radiated from a monopole, IRE Trans. Ant. Prop. AP-4, 179.

Wait, J. R. (1959), Transmission of power in radio propagation, Electronic and Radio Engineer 36, 146.

Wait, J. R., and H. H. Howe (1956), Amplitude and phase curves for ground-wave propagation in the band 200 cycles per second to 500 kilocycles, NBS Circ. 574.

Wait, J. R., and W. J. Surtees (1954), Impedance of a toploaded antenna of arbitrary length over a circular grounded screen, J. Appl. Phys. 25, 553.

(Paper 68D7-379) 